\title{
Chapter 4 \\ CEAR: Cluster based Energy Aware Routing Algorithm to Maximize Lifetime of Wireless Sensor Networks (WSNs)
}

\author{
H. Sivasankari, R. Leelavathi, M. Vallabh, K. R. Venugopal, \\ S. S. Iyengar and L. M. Patnaik
}

\begin{abstract}
Technological development in wireless communication enables the development of smart, tiny, low cost and low power sensor nodes to outperform for various applications in Wireless Sensor Networks. In the existing Tabu search algorithm, clusters are formed using initial solution algorithm to conserve energy. We propose a Cluster Based Energy Aware Routing (CEAR) algorithm to maximize energy conservation and lifetime of network with active and sleep nodes. The proposed algorithm, removes duplication of data through aggregation at the cluster heads with active and sleep modes. A comparative study of CEAR algorithm with Tabu search algorithm is obtained. Comparative study shows improvement in the Lifetime and energy conservation by 17 and $22 \%$ respectively over the existing algorithm.
\end{abstract}

Keywords Clusters $\cdot$ Delay $\cdot$ Energy aware routing $\cdot$ Lifetime and wireless sensor networks

H. Sivasankari $(\bowtie) \cdot R$. Leelavathi $\cdot$ M. Vallabh $\cdot$ K. R. Venugopal

Department of Computer Science and Engineering, University Visvesvaraya College of

Engineering, Bangalore University, Bangalore 560 001, India

e-mail: sankari@yahoo.com

S. S. Iyengar

Florida International University, Miami, USA

L. M. Patnaik

Indian Institute of Science, Bangalore, India 


\subsection{Introduction}

Wireless Sensor Networks (WSNs) are deployed with hundreds or thousands of computable and low cost sensors. These sensor nodes are multi-functional and with computing capabilities. These sensor nodes are integrated on a single board in a few cubic inches with embedded microprocessor, radio receivers, sensing, and computing and communication unit. They are powered by $50 \mathrm{~W}$ and can last for 2-3 years with very less duty cycling and these sensor nodes are prone for failures. In WSN there are three types of communications, they are clock driven, event driven and query driven. In the clock driven approach, data collection and transmission takes place at regular periodic intervals. In the event driven and query driven approaches, data collection is triggered by events or queries. Data Aggregation, Clustering, effective routing and data compression, min-max and averaging methods are used to reduce energy consumption in WSNs. Tabu search algorithm [1] form clusters of sensor nodes and routes data from source to the destination through cluster heads. The sensor nodes are active and transmits data without aggregation all the time and this approach consumes more energy as there is no aggregation. We propose Cluster based Energy Aware Routing (CEAR) in order to maximize lifetime and energy conservation of WSNs. In a cluster, sensor node works in active and sleep mode randomly. It routes the data from source to destination through cluster head with aggregation. Thus, it reduces the energy consumption for sending the large set of data. The rest of the paper is organized as follows: Related work is discussed in Sect. 4.2. Problem Definition is formulated in Sect. 4.3. Algorithm is developed in Sect. 4.4. Simulation and Performance Analysis are analyzed in Sect. 4.5. Conclusions are presented in Sect. 4.6.

\subsection{Literature Survey}

Heinzelman et al. [2] developed a Low Energy Adaptive Clustering Hierarchy (LEACH) algorithm for clustering. LEACH is a distributed approach and it requires no control information from the base station. In this approach data is required to be sent in the allotted time. Madan et al. [3] proposed TAG: Tiny Aggregation Service for Ad-Hoc Sensor networks. Interface for data collection, aggregation, executed aggregation queries in the sensor networks. Aggregation queries are executed in time, efficiently. Each mote is required to transmit only a single message per epoch, regardless of its depth in the routing tree. It is sensitive to resource constraints and lossy communication. Liang et al. [4] have designed a genetic cost model for data gathering in sensor networks, as routing tree used for query evaluation and therefore the network is balanced. The proposed heuristic algorithm showed that online gathering problem is NP complete. It is focused only on the event driven data and data gathering queries are assumed sequential. Ghiasi et al. [5] proposed a balanced $\mathrm{K}$ clustering algorithm to minimize energy 
consumption using minimum cost network flow. Agarwal et al. [6] have proposed sub exponential algorithms to solve the K-center problem to compute the optimal K-center. They considered the metrics for planar case, i.e., smaller dimensions. Noritaka et al. [7] have proposed centralized and distributed approaches for Clustering. These methods prolong the network lifetime than the conventional methods.

\subsection{Problem Definition and Mathematical Model}

Given a set of Wireless Sensor Nodes $S_{i} \in V$ where $i=1,2, \ldots, n$. we consider a single sink with maximum coverage radio and power. The objectives are to reduce the energy consumption and to maximize the lifetime of the network. The assumptions are (i) All source sensor nodes are static. (ii) Links in between the nodes are bidirectional. (iii) Sink has long communication range and energy than the source sensor nodes (iv) The highest energy node becomes the cluster head. (v)Sensor nodes act in either active or sleep mode.

\subsubsection{Mathematical Model}

All sensor nodes are deployed with equal amount of energy. Clusters are formed based on Euclidean distances. Cluster size is increased as the number of node increases in the deployment. Graph $(\mathrm{G})$ i.e., $\mathrm{G}=(\mathrm{V}, \mathrm{E})$ where $\mathrm{V}$ is a set of vertices and $\mathrm{E}$ is a set of edges. Neighbor of a node is selected based on the following constraints. In a graph, $v_{i}$ is the source node and $v_{j}$ next neighbor node to receive data as the destination. The distance between source and destination is calculated based on Euclidean distance.

$$
\begin{gathered}
\text { Dist }\left(v_{i}-v_{j}\right)<\text { Range } \\
\text { Euclidean Distance }=\sqrt{\left(x_{i}-x_{j}\right)^{2}+\left(y_{i}-y_{j}\right)^{2}} \\
\text { List of Neighbors } L\left(N_{i}\right)=\bigcup_{V_{j} \in V}\left\{V_{j} / \operatorname{Dist}\left(v_{j}, v_{i}\right)<r\right\}
\end{gathered}
$$

Theorem Aggregation at the cluster head removes duplication of data thus it increases energy conservation and lifetime.

$$
C(e)=\sum_{i=1}^{n}\left(C_{i}\right)\left(n_{i}\right)
$$




$$
\begin{gathered}
A(e)=l(v)\left(1-\sigma_{u v} X_{e}\right) \\
P_{\text {total }}=T(e)+A(e)+R(e)+C(e)
\end{gathered}
$$

Where $T(e)$ is Transmission cost, $A(e)$ is Aggregation cost, $\sigma_{\mathrm{uv}}$ is reduction ratio and $X_{e}$ is the Aggregation factor if $X_{e}=1$ there is an aggregation else there is no aggregation, $R(e)$ Reception cost, $C(e)$ Cluster formation cost, load on node $v, C_{i}$ represents the ith cluster and $n_{i}$ is the number of nodes in a cluster.

\subsection{CEAR: Cluster Based Energy Aware Routing Algorithm}

In a Cluster based Energy Aware Routing (CEAR), clusters are formed as the nodes are deployed in the region. When the first node is deployed, the cluster is formed and it becomes the cluster head. Later, the deployed nodes identify the neighboring nodes by sending hello packets. When it receives successive echo packets from neighboring nodes then it is added to the cluster. If the node is neither in the range nor in neighbor list of any cluster then a new cluster is formed and node becomes the cluster head. As the more and more nodes are deployed, the cluster size is increased. This procedure is repeated until there is no more node to be deployed.

In a cluster, after each iteration it checks for the highest energy node. It elects the highest energy node as the cluster head. In a cluster, a set of source nodes act in active and sleep mode. Before a node goes for sleep mode it ensures that enough number of nodes are in active mode in order to ensure that any event during the transition of active and sleep mode is not missed. Energy Aware Routing helps the data to be routed through cluster heads (Table4.1).

\subsection{Simulation and Performance Analysis}

In the setup of MATLAB simulation, a $100 \times 100 \mathrm{~m}$ region is considered with 150 sensor nodes. All sensor nodes have equal amount of energy initially with $50 \mathrm{~J}$. Radio model is lossy in nature and communication range is $150 \mathrm{~m}$, energy consumption per bit is $60 \mathrm{pJ}$. Control packet size is 500 bytes. Fig 4.1 shows the graph between the energy consumption with number of sensor nodes or clusters. The proposed algorithm CEAR consumes lower energy than the existing algorithm Tabu. The energy savings in CEAR is $22 \%$ higher than the existing Tabu search algorithm. Fig 4.2 depicts that the lifetime is increased by $17 \%$ in CEAR algorithm in comparison with the existing algorithm. 
Table 4.1 Cluster based energy aware routing algorithm

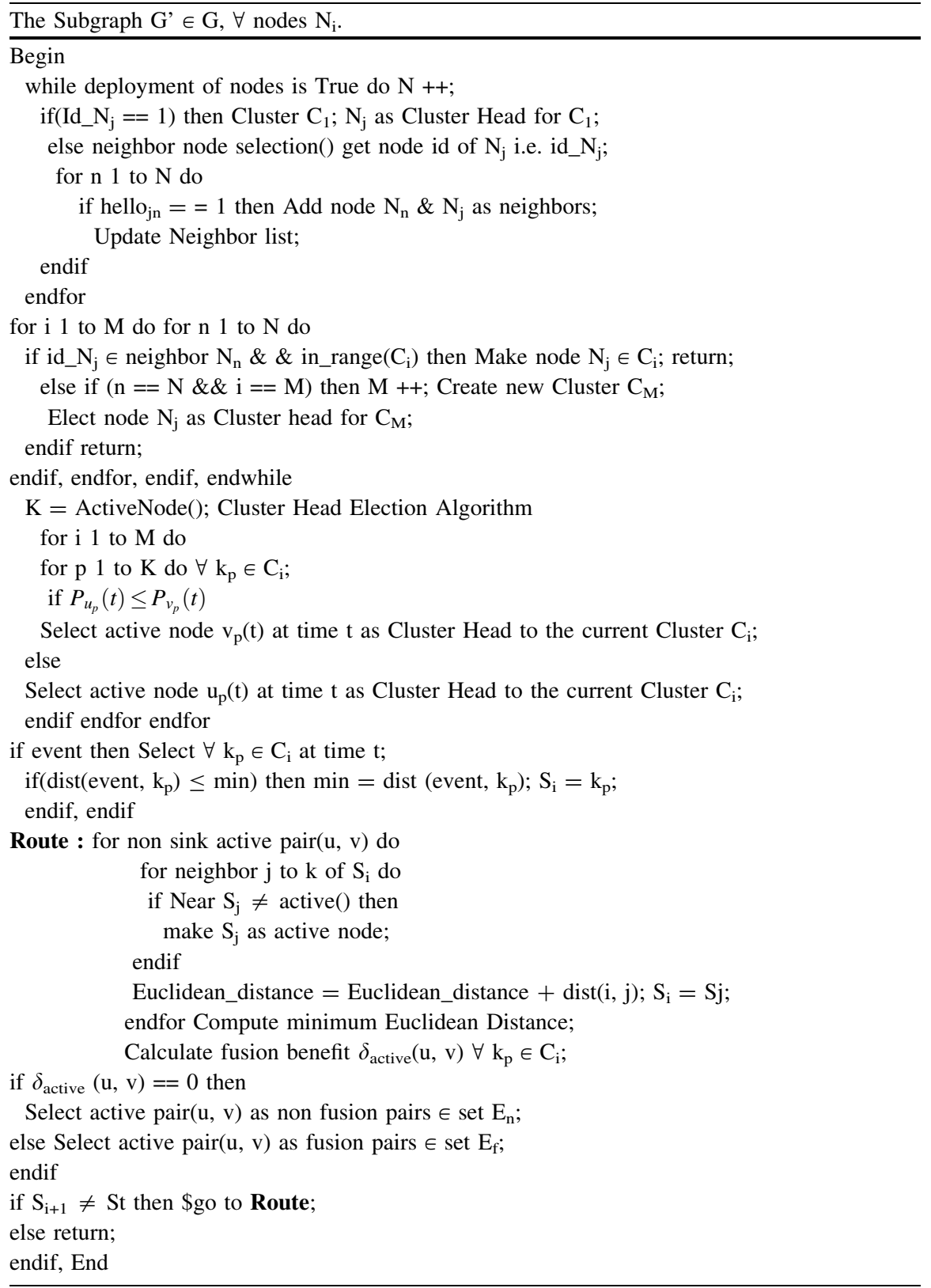


Fig. 4.1 Lifetime

Comparison between Tabu Search and CEAR

Fig. 4.2 Energy consumption versus number of cluster heads in routing path
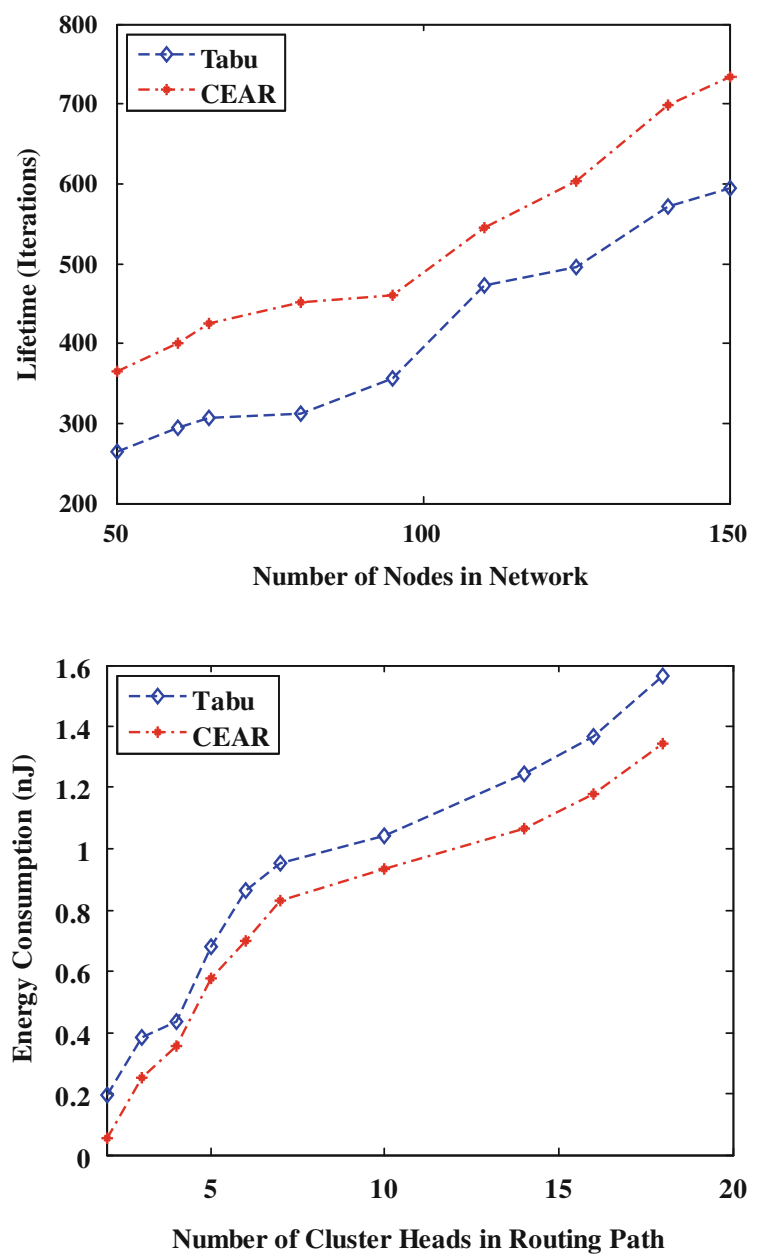

\subsection{Conclusions}

Wireless sensor networks works with limited battery and lifetime. We propose Cluster based Energy Aware Routing (CEAR) to conserve energy and maximize the life time of the WSNs. In the case of Tabu search algorithm, clusters are formed and routed through clusters heads without aggregation and active/sleep modes. Therefore, it consumes more energy. The data aggregation and periodic active/sleep mode in CEAR algorithm improves the life time and energy conservation, This work can be enhanced in future to reduce delay. 


\section{References}

1. El Rhazi A, Pierre S (2009) A Tabu search algorithm for cluster building in wireless sensor networks. IEEE Trans Wirel Comput 8(4):433-444

2. Heinzelman W, Chandrakasan A, Balakrishnan H (2002) An application specific protocol architecture for wireless microsensor networks. IEEE Trans Wirel Commun 1(4):660-670

3. Madden SR, Franklin MJ, Hellerstein JM, Hong W (2002) TAG: tiny aggregation service for ad-hoc sensor networks. In: Proceedings of the fifth symposium operating systems design and implementation (OSDI 02), pp 131-146

4. Liang W, Liu Y. (2007) Online data gathering for maximizing network lifetime in sensor networks, IEEE Trans Mobile Comput 6(1):2-11

5. Ghiasi S, Srivastava A, Yang X, Sarrafzadeh M (2002) Optimal energy aware clustering in sensor networks. IEEE Trans Mobile Comput 2:258-269

6. Agarwal PK, Procopiuc CM (2002) Exact and approximation algorithms for clustering. Algorithmica 33(2)201-226

7. Shigei N, Miyajima H, Morishita H, Marda M (2009) Centralized and distributed clustering method for energy efficient wireless sensor networks. In: Proceedings of the International Multiconference of Engineers and Computer Scientists(IMECS), Vol.2174, No 1, LNCS, pp $423-427$ 